\title{
Tenapanor: A New Treatment Option for Hyperphosphatemia in End Stage Kidney Disease
}

\author{
Tiffany Lin ${ }^{1}$, Akram Al-Makki², Brian Shepler ${ }^{1}$ \\ ${ }^{1}$ Department of Pharmacy Practice, Purdue University College of Pharmacy, West Lafayette, IN, USA; ${ }^{2}$ IU Health Arnett \\ Nephrology, Lafayette, IN, USA
}

Corresponding author: Brian Shepler, PharmD., Purdue University College of Pharmacy, 575 Stadium Mall Drive, West Lafayette, IN 47907-2091, United States; TEL: (765)-494-1365; email: sheplerb@purdue.edu

Received, September 24, 2021; Revised, January 13, 2022; Accepted January 14, 2022; Published, January 18, 2022

\begin{abstract}
Purpose: This narrative review explores the currently published studies that have evaluated tenapanor for the treatment of hyperphosphatemia in end-stage kidney disease (ESKD) patients on hemodialysis. This medication's new phosphate lowering mechanism of action reduces intestinal phosphate absorption predominantly through reduction of passive paracellular phosphate flux by inhibition of the sodium/hydrogen exporter isoform 3 (NHE3). Tenapanor additionally prevents active transcellular phosphate absorption compensation by decreasing the expression of sodium phosphorus $2 \mathrm{~b}$ transport protein (NaPi2b). Methods: A comprehensive search of the literature was conducted using PubMed and ClinicalTrials.gov search engines. The search term "tenapanor hyperphosphatemia" was used for study retrieval. Results were limited to studies published in the English language and excluded review articles. Human, animal, and in vitro studies were included. No date range was specified. Results: A total of 11 primary studies were identified and included in this review, the largest human study of which enrolled 236 patients. Each study is presented in table format along with measured end points. Conclusions: Tenapanor is the first drug in its class that lowers hyperphosphatemia in ESKD patients through a novel mechanism of action involving paracellular inactive transport. Although more studies are needed, early results indicate that tenapanor may have a place in managing hyperphosphatemia in ESKD patients both as monotherapy and as an adjunct to existing phosphate binder therapy.
\end{abstract}

\section{INTRODUCTION}

Hyperphosphatemia is a significant complication that plagues nearly all patients with late-stage chronic kidney disease (CKD) and end-stage kidney disease (ESKD) (1). This is a consequence of the deteriorating kidney's inability to excrete phosphorus from the body thereby allowing dietary phosphorus absorption and serum accumulation. Hyperphosphatemia in ESKD can lead to secondary hyperparathyroidism, renal osteodystrophy, metastatic calcification, and cardiovascular mortality. In addition to dietary restriction of phosphorus and hemodialysis, drugs that bind phosphate in the gut are usually needed as well (2). The Kidney Disease Improving Global Outcomes' (KDIGO) latest hyperphosphatemia guidelines recommend treating hyperphosphatemia by limiting phosphorus intake from the diet and using phosphate-binding medications (1). Phosphate binders continue to be a mainstay of treatment for hyperphosphatemia and exist in several different categories including the oldest calcium-containing binders (calcium carbonate, calcium acetate), the polymer-based binder (sevelamer carbonate), the metal-based binders (lanthanum carbonate, aluminum hydroxide) and the newest additions to the armamentarium, the iron-based binders (sucroferric oxyhydroxide, ferric citrate) (3). These medications all control phosphorus absorption by binding to dietary phosphorus in the gastrointestinal tract and allowing the chelate to pass from the body into the feces. Aluminum hydroxide is no longer recommended for long-term use because of concerns about its toxicity, and calcium citrate should be avoided in all CKD patients since citrate can markedly increase intestinal aluminum absorption $(4,5)$.

Recent developments in the understanding of how phosphorus is absorbed from a mechanistic standpoint have allowed innovative new therapies to be conceptualized. From animal model studies, two distinct mechanisms for phosphorus absorption have been identified. Active transcellular transport 
through specific ion transporters and inactive paracellular transport which occurs through tight junctions of cells in the duodenum are new biologic pathways for phosphorus absorption from the gastrointestinal tract (6). Active transcellular transport is facilitated through the sodium phosphorus $2 \mathrm{~b}$ transport protein $(\mathrm{NaPi} 2 \mathrm{~b})$ and is located on the apical side the of the intestinal lumen. This protein is expressed when low serum phosphorus increases parathyroid hormone (PTH) concentrations which then stimulates production of activated vitamin D3. Similarly, high serum phosphorus concentrations increase fibroblast growth factor 23 (FGF23) concentrations and lower PTH concentrations which reduce active vitamin D3 production and decrease NaPi2b production (7). Paracellular phosphorus absorption is an inactive transport that is mediated by the sodium/hydrogen exporter isoform 3 (NHE3) protein. This is thought to be a more potent controller of phosphorus absorption and a better target for directed drug therapy (8).

The focus of this review and the novelty of this work is on one such drug therapy product, the first novel NHE3 inhibitor to be studied in human subjects for the treatment of hyperphosphatemia, tenapanor hydrochloride. Tenapanor, a minimally systemically absorbed molecule, is an inhibitor of intestinal NHE3 that was developed to treat irritable bowel syndrome with constipation but that also lowers serum phosphorus by blocking its paracellular transport from the intestinal lumen. Tenapanor reduces intestinal phosphate absorption predominantly through reduction of passive paracellular phosphate flux. Tenapanor also prevents active transcellular phosphate absorption compensation by decreasing the expression of $\mathrm{NaPi} 2 \mathrm{~b}(9)$.

\section{METHODS}

To complete this narrative review, a search of the literature was conducted using PubMed and ClinicalTrials.gov search engines. The search term "tenapanor hyperphosphatemia" was used for both search engines. Results were limited to studies published in the English language and excluded review articles. Human, animal, and in vitro studies were included. No date range was specified.

\section{RESULTS}

Using the search methods described above, 17 articles were returned. After excluding review articles, 11 primary studies were identified. All phase one through phase three articles were included in our review for completeness. Another 11 studies are currently recruiting patients, underway, or do not have published results. Of the published trials, the largest study in humans enrolled 236 patients. Most studies evaluated the efficacy of tenapanor for hyperphosphatemia while others focused on drugdrug interactions. Table 1 depicts a summary of the details and findings obtained from the described search method.

\section{DISCUSSION}

Phase one trials were included in this review to thoroughly examine tenapanor's effect on hyperphosphatemia and potential drug-drug interactions. These drug-drug interactions are a crucial part of clinical practice and in the case of tenapanor involve CYP450 inhibition, phosphate binders, and PepT1. All three drug interaction studies in vivo concluded that there was no difference in serum concentrations of the test drug and tenapanor when both were co-administered (10-12). It is important to note that there is no drug-drug interaction between phosphate binders and tenapanor, as dual therapy with these agents may be warranted with tenapanor in patients with persistent hyperphosphatemia.

A few publications included data from in vitro, animal, and human studies together. Some outcomes from the in vitro and animal studies in those select publications did not translate into the same results in vivo. For example, one study considered that tenapanor, although minimally systemically absorbed, may exhibit drug-drug interactions with CYP450 enzymes since CYP3A4 is also present in the cells of the gut wall. In vitro studies displayed inhibition of CYP3A4 by tenapanor. However, no such interaction was observed from the coadministration of midazolam (a model substrate of CYP3A4) and tenapanor in human studies (10). This phenomenon could possibly be due to a higher concentration of tenapanor used in vitro compared to that found in human intestinal tissue.

All the phase three trials had a primary endpoint of change in serum phosphorus concentrations from baseline in patients suffering from hyperphosphatemia. The proportion of patients that reached a serum phosphorus concentration of < $5.5 \mathrm{mg} / \mathrm{dL}$ were only included as a secondary endpoint $(13,14)$. Normal serum phosphorus concentrations are between 2.5 and $5.5 \mathrm{mg} / \mathrm{dL}$. 
Table 1. Summary of In Vitro, Animal, and Human Studies for Tenapanor

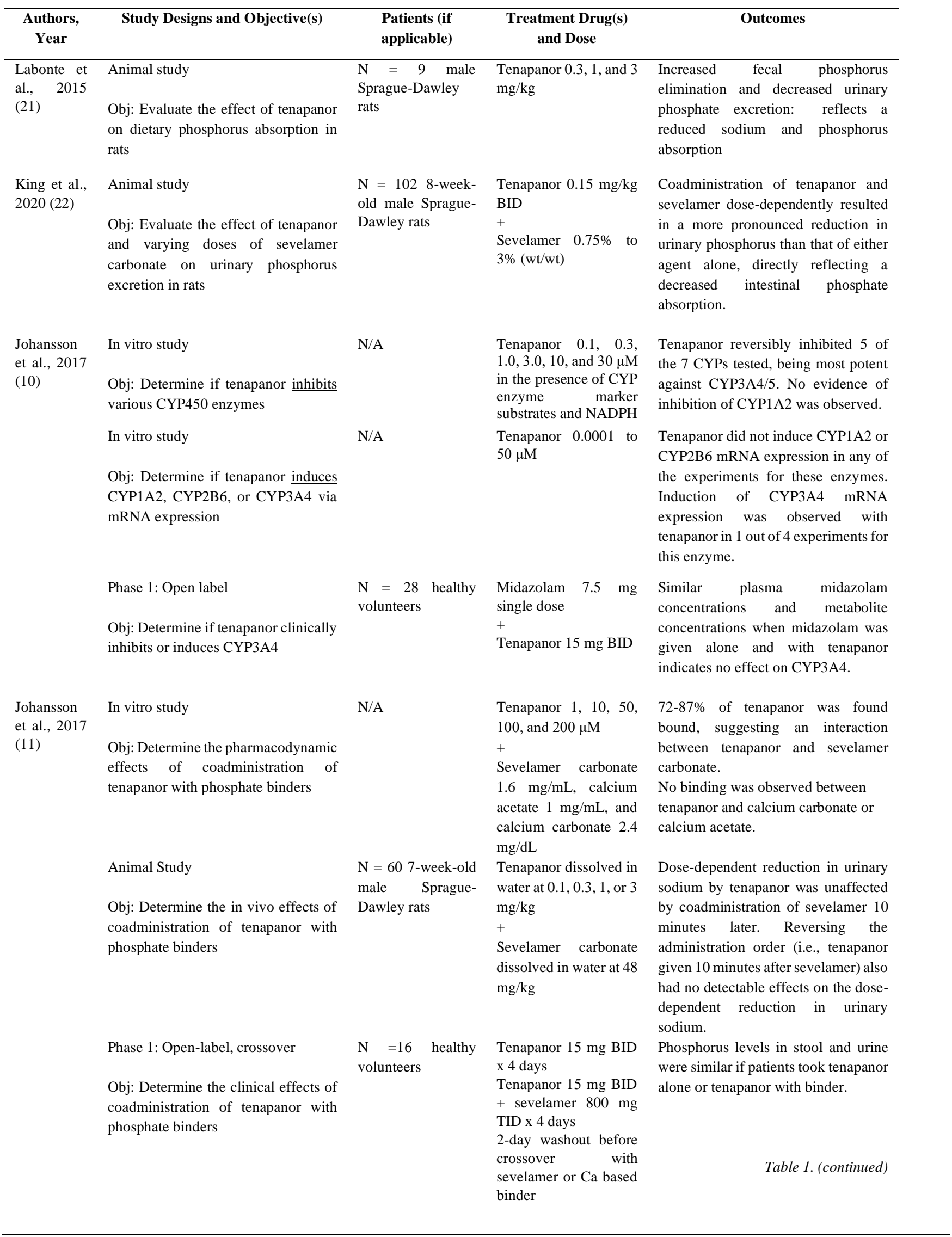




\section{Johansson Phase 1: Open-label, crossover \\ et al., 2017 \\ Obj: Determine tenapanor's effect on \\ PepT1 activity in healthy volunteers}

$\mathrm{N}=28$ healthy volunteers
$\mathrm{N}=83$ healthy adults

Obj: To report on the safety and efficacy of tenapanor on healthy subjects

King et al., Animal Studies

2018 (8)

Block et al., 2017 (16)

Block et al., Phase 2b: RCT

2019 (24)
Obj: Evaluate the effect of tenapanor on serum FGF23

Obj: Determine the mechanism by which tenapanor reduces gastrointestinal phosphate uptake

\section{Phase 1: Open-label, crossover}

Obj: Determine the effect of tenapanor on intestinal phosphate absorption

Phase 2: RCT, dose-ranging

Obj: Evaluate the effect of tenapanor on serum phosphate concentration in HD patients with hyperphosphatemia

$\mathrm{N}=162$

CKD stage 5D

adults on

maintenance HD

and with

hyperphosphatemia

$\mathrm{N}=162$ CKD stage 5D patients receiving $\mathrm{HD}$ on stable dose of phosphate binders with hyperphosphatemia
Single dose cefadroxil $500 \mathrm{mg}$ in AM for 1 day

$+$

Tenapanor $15 \mathrm{mg}$ BID $x 4$ days, followed by single doses of both tenapanor $15 \mathrm{mg}$ and cefadroxil $500 \mathrm{mg}$ on day 5

4-day washout between treatments

Cefadroxil plasma concentration-time curves were similar whether cefadroxil was administered alone or in combination with tenapanor. PK parameters also similar when cefadroxil was given alone or with tenapanor indicating no effect on PepT1's absorption activity.

Tenapanor $180 \quad \mathrm{mg}$ single dose or 15,30 , 60 , or $90 \mathrm{mg}$ BID x 7 days

Diarrhea and abdominal pain were the most common AEs related to treatment.

Repeated doses of tenapanor resulted in increased stool phosphorus and decreased urine phosphorus.

All tenapanor doses

Table 1. (continued)

resulted in plasma concentrations below lower limit of quantification suggesting low systemic absorption.

Various tenapanor Tenapanor produces its effect by tightdoses based on the junction modulation which increases experiment performed transepithelial electrical resistance and reduces permeability to phosphate, reducing paracellular phosphate absorption.

Tenapanor prevents active transcellular phosphate transport by decreasing NaPi2b expression.

Tenapanor $15 \mathrm{mg}$ BID $\mathrm{x} 4$ days

Significantly increased mean daily stool phosphorus excretion and significantly decreased mean daily urinary phosphorus excretion.

Significantly reduced mean daily urinary sodium excretion.

No effect on potassium excretion.

Tenapanor 3 or $30 \mathrm{mg}$

Statistically significant dosedependent reduction in serum phosphate with largest reductions in the tenapanor 10 and $30 \mathrm{mg}$ BID groups

No significant difference in serum PTH

Tenapanor 3 or $30 \mathrm{mg}$ Tenapanor treatment decreased serum daily; or 1,3, 10, or 30 FGF concentrations from postmg twice daily phosphate binder washout. FGF23 continued to rise in patients receiving placebo after washout.

Table 1. (continued) 


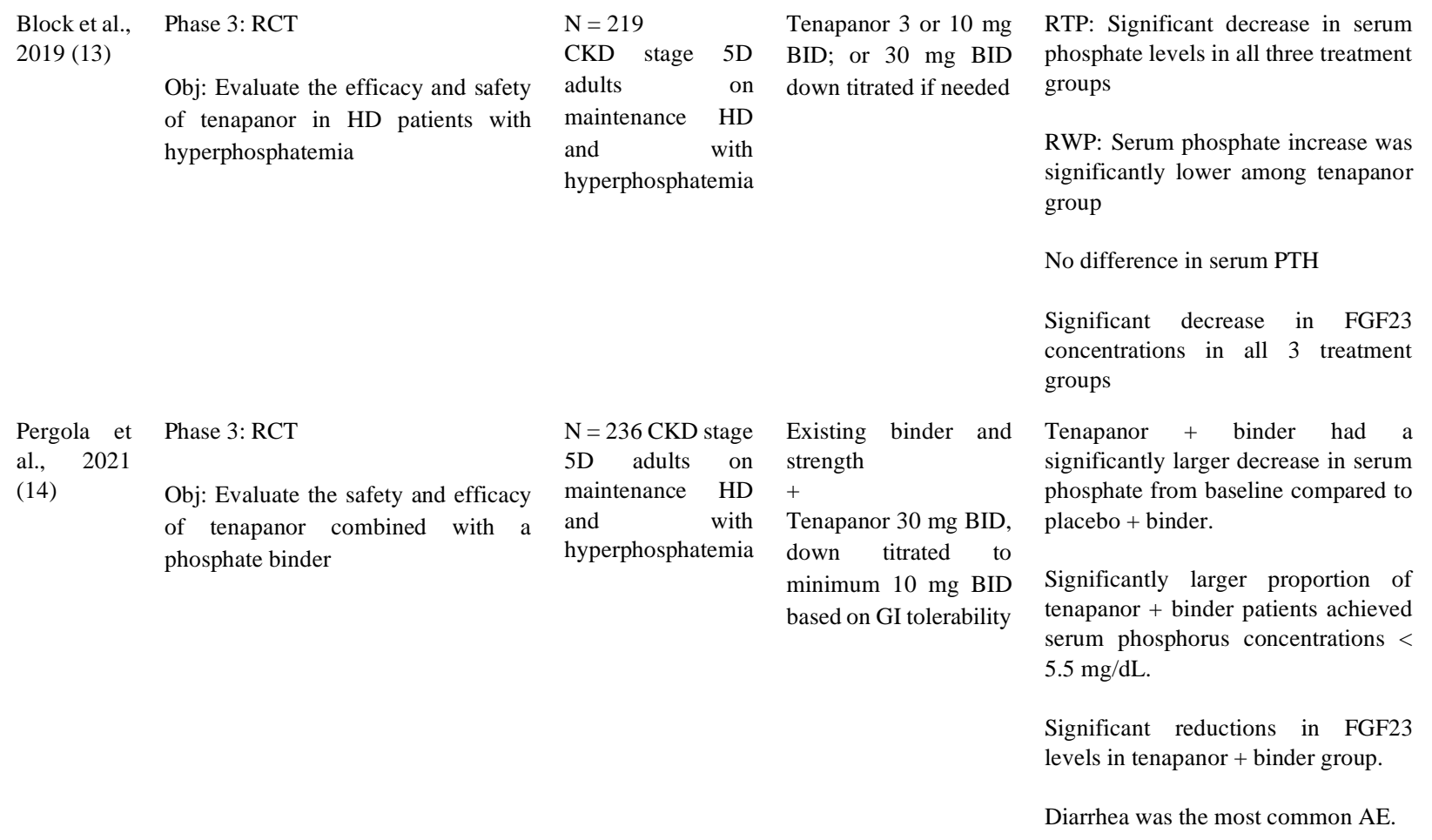

BID: twice daily; TID: three times daily; PK: pharmacokinetic; RCT: randomized controlled trial; AE: adverse event; HD: hemodialysis; RTP: randomized treatment period; RWP: randomized withdrawal period

Reducing a patient's serum phosphorus levels to $<$ $5.5 \mathrm{mg} / \mathrm{dL}$ would be what is clinically significant rather than merely recognizing a change in serum phosphorus from baseline. The ongoing NORMALIZE (NCT03988920) trial is studying this clinical significance, as its primary endpoint is the proportion of patients who reach a normal serum phosphorus concentration (2.5-5.5 mg/dL) (15).

Only one clinical study, AMPLIFY, has tested the combination of tenapanor with phosphate binder therapies. Phosphate binders tested included sevelamer and non-sevelamer binders. The study investigators did not include which binders comprised the non-sevelamer group. Tenapanor + binder resulted in a significantly larger decrease in serum phosphorus compared to placebo + binder (0.84 vs. $-0.19 \mathrm{mg} / \mathrm{dL} ; \mathrm{P}<0.001)(14)$. No tenapanor monotherapy group was included in the study design and no definitive statement can be made regarding the additive or synergistic effect of tenapanor. It is likely that given the complementary mechanism of action that some degree of synergy may exist.

All clinical trials in human subjects published to date have been conducted in ESKD patients receiving maintenance hemodialysis. There is evidence that tenapanor as monotherapy as well as given concomitantly with phosphate binders lowers serum phosphorus levels. Hemodialysis patients currently taking phosphate binders with uncontrolled hyperphosphatemia may benefit from tenapanor to reduce the pill burden of phosphate binders. Adherence with phosphate binders was low at 38\% and phosphate binders accounted for half of a patient's total pill burden (16). $70 \%$ of patients on hemodialysis continued to experience elevated serum phosphorus despite efforts to control levels through diet and phosphate binder therapy (14). Tenapanor has been dosed between 10-30 mg twice daily without regard to meals in the studies presented here. This dosing schedule and pill burden could be considerably less than those for phosphate binders since phosphate binders require administration three times daily with meals. This drug's twice daily dosing can be a benefit for patients suffering from excessive pill burden. Although not specifically studied in humans, cardiovascular calcification would not be expected with tenapanor compared to other calcium-containing phosphate binders such as calcium carbonate as it does not contain calcium.

From the evidence presented, it appears tenapanor as an adjunct therapy to phosphate binders produces the greatest decrease in serum phosphorus concentrations (14). Monotherapy with tenapanor also lowers serum phosphorus although not to the same degree as dual therapy. The ongoing NORMALIZE trial is evaluating the ability of 
tenapanor alone or in combination with sevelamer to achieve normal serum phosphorus concentrations in patients with ESKD (15). Once published, this information will further guide clinicians in the appropriate use of tenapanor.

It is important for prescribers to know and share with patients that diarrhea (16\%), flatulence, and abdominal distention (3\% each) have been the most common adverse effects of tenapanor. The drug is contraindicated for use in children $<6$ years old and in patients with intestinal obstruction (17-19).

In July 2021, the Food and Drug Administration (FDA) issued a Complete Response Letter to the pharmaceutical company Ardelyx, the makers of tenapanor, and stated that more significant clinical data was needed before approval could be granted for the indication of hyperphosphatemia. Although the current data does show a reduction in phosphorus serum concentrations, the magnitude of this effect requires more investigation (20).

\section{CONCLUSION}

In conclusion, tenapanor has shown a unique mechanism of action to reduce phosphate absorption from the intestines, thus further reducing serum phosphorus concentrations in patients. Published data to date show efficacy as monotherapy and a greater effect when combined with phosphate binders. When used as monotherapy, this may reduce excessive pill burden from phosphate binders. No significant drug interactions have been observed clinically, and tenapanor can be administered without regard to meals. The long-term safety of tenapanor is unknown as well as its efficacy in CKD patients who are not on hemodialysis. More large, randomized trials regarding the use of tenapanor, powered for cardiovascular and/or mortality outcomes, in CKD as well as ESKD patients, are needed, especially for subpopulations that have not been adequately represented in previous trials. In addition, more data demonstrating the magnitude of the phosphorus lowering effect will be needed before FDA approval is granted to tenapanor for treating hyperphosphatemia.

\section{ACKNOWLEDGEMENTS}

The authors have nothing to disclose regarding the preparation of this work and have not received financial support of any kind.

\section{REFERENCES}

1. KDIGO 2017 Clinical Practice Guideline Update for the Diagnosis, Evaluation, Prevention, and Treatment of Chronic Kidney Disease - Mineral and Bone Disorder (CKDMBD). Kid Int Suppl. 2017 Jul;7(1):1-59. DOI: 10.1016/j.kisu.2017.04.001

2. Phosphate Binders. Med Lett Drugs Ther. 2006 Feb;48(1228):15-6.

3. Floego J. Phosphate binders in chronic kidney disease: a systematic review of recent data. $\mathbf{J}$ Nephrol. 2016 Jun;29(3):329-40. DOI: 10.1007/s40620-016-0266-9

4. Molitoris BA, Froment DH, Mackenzie TA, Huffer WH, Alfrey AC. Citrate: a major factor in the toxicity of orally administered aluminum compounds. Kidney Int. 1989 Dec;36(6):949-53. DOI: 10.1038/ki.1989.286

5. Nolan CR, Califano JR, Butzin CA. Influence of calcium acetate or calcium citrate on intestinal aluminum absorption. Kidney Int. $1990 \quad$ Nov;38(5):937-41. DOI: 10.1038/ki.1990.294.

6. Fouque D, Vervloet M, Ketteler M. Targeting gastrointestinal transport proteins to control hyperphosphatemia in chronic kidney disease. Drugs. 2018 Aug;78(12):1171-86. DOI: 10.1007/s40265-018-0950-2

7. Cozzolino M, Ketteler M, Wagner CA. An expert update on novel therapeutic targets for hyperphosphatemia in chronic kidney disease: preclinical and clinical innovations. Expert Opin Ther Targets. 2020 May;24(5):477-88. DOI: 10.1080/14728222.2020.1743680

8. King AJ, Siegel M, He Y, et al. Inhibition of sodium/hydrogen exchanger 3 in the gastrointestinal tract by tenapanor reduces paracellular phosphate permeability. Sci Transl Med. 2018 Aug;10(456):eaam6474. DOI: 10.1126/scitranslmed.aam6474

9. Saurette M, Alexander RT. Intestinal phosphate absorption: the paracellular pathway predominates? Exp Biol Med (Maywood). 2019 May;244(8):646-54. DOI: $10.1177 / 1535370219831220$

10. Johansson S, Rosenbaum DP, Ahlqvist M, et al. Effects of tenapanor on cytochrome P450mediated drug-drug interactions. Clin Pharmacol Drug Dev. 2017 Sep;6(5):466-75. DOI: $10.1002 /$ cpdd. 346 
11. Johansson S, Leonsson-Zachrisson $\mathrm{M}$, Knutsson M, et al. Preclinical and healthy volunteer studies of potential drug-drug interactions between phosphate binders. Clin Pharmacol Drug Dev. 2017 Sep;6(5):448-56. DOI: $10.1002 /$ cpdd. 307

12. Johansson S, Rosenbaum DP, Palm J, et al. Tenapanor administration and the activity of the $\mathrm{H}^{+}$-coupled transporter PepT1 in healthy volunteers. $\mathrm{Br}$ J Clin Pharmacol. 2017 Sep;83(9):2008-14. DOI: 10.1111/bcp.13313

13. Block GA, Rosenbaum DP, Yan A, Chertow GM. Efficacy and safety of tenapanor in patients with hyperphosphatemia receiving maintenance hemodialysis: a randomized phase 3 trial. J Am Soc Nephrol. 2019 Apr;30(4):641-52.

DOI: 10.1681/ASN.2018080832

14. Pergola PE, Rosenbaum DP, Yang Y, Chertow GM. A randomized trial of tenapanor and phosphate binders as a dual-mechanism treatment for hyperphosphatemia in patients on maintenance dialysis (AMPLIFY). J Am Soc Nephrol. 2021 Jun;32(6):1465-73. DOI: 10.1681/ASN.2020101398

15. NIH U.S. National Library of Medicine. A long-term study to evaluate the ability of tenapanor alone or in combination with sevelamer to treat to goal serum phosphorus in patients with end-stage kidney disease on dialysis (NORMALIZE). Accessed June 8, 2021.

https://clinicaltrials.gov/ct2/show/NCT03 988920?term=nct03988920\&draw $=2 \&$ ra $\mathrm{nk}=1$

16. Block GA, Rosenbaum DP, LeonssonZachrisson M, et al. Effect of tenapanor on serum phosphate in patients receiving hemodialysis. J Am Soc Nephrol. 2017 Jun;28(6):1933-42.

DOI: 10.1681/ASN.2016080855

17. Munjal A, Dedania B, Cash B. Update on pharmacotherapy for irritable bowel syndrome. Curr Gastroenterol Rep. 2019 Apr;21(6):25. DOI: 10.1007/s11894-0190692-7

18. Chey WD, Lembo AJ, and Rosenbaum DP. Efficacy of tenapanor in treating patients with irritable bowel syndrome with constipation: a 12-week, placebo-controlled phase 3 trial (T3MPO-1). Am J Gastroenterol 2020
Feb;115(2):281-293.

DOI: 10.14309/ajg.0000000000000516

19. Drugs for Irritable Bowel Syndrome. Med Lett Drugs Ther. 2020 Mar;62(1594):41-8.

20. Melville NA. Medscape. FDA Rejects tenapanor for control of phosphorus in kidney disease. Accessed October 12, 2021. https://www.medscape.com/viewarticle/ 956066

21. Labonte ED, Carreras CW, Leadbetter MR, et al. Gastrointestinal inhibition of sodiumhydrogen exchanger 3 reduces phosphorus absorption and protects against vascular calcification in CKD. J Am Soc Nephrol. 2015 May;26(5):1138-49.

DOI: 10.1681/ASN.2014030317

22. King AJ, Kohler J, Fung C, et al. Combination treatment with tenapanor and sevelamer synergistically reduces urinary phosphorus excretion in rats. Am J Physiol Renal Physiol. 2021 Jan 1;320(1):F133-F144. DOI: 10.1152/ajprenal.00137.2020

23. Johansson S, Rosenbaum DP, Knutsson M, Leonsson-Zachrisson, M. A phase 1 study of the safety, tolerability, pharmacodynamics and pharmacokinetics of tenapanor in healthy Japanese volunteers. Clin Exp Nephrol. 2017 Jun;21(3):407-16. DOI: 10.1007/s10157-016$1302-8$

24. Block GA, Rosenbaum DP, Yan A, Greasley PJ, Chertow GM, Wolf M. The effects of tenapanor on serum fibroblast growth factor 23 in patients receiving hemodialysis with hyperphosphatemia. Nephrol Dial Trans. 2019 Feb;34(2):339-46. DOI: 10.1093/ndt/gfy061 